\title{
Elective affinities: a Tudor-Aubergine tale of germline partnership
}

\author{
Anastassios Vourekas, Yohei Kirino, and Zissimos Mourelatos ${ }^{1}$ \\ Department of Pathology and Laboratory Medicine, Division of Neuropathology, University of Pennsylvania School of Medicine, \\ Philadelphia, Pennsylvania 19104, USA
}

\begin{abstract}
In Drosophila melanogaster and many other metazoans, the specification of germ cells requires cytoplasmic inheritance of maternally synthesized RNA and protein determinants, which are assembled in electron-dense cytoplasmic structures known as germ or polar granules, found at the posterior end of the oocytes. Recent studies have shown that the formation of germ granules is dependent on the interaction of proteins containing tudor domains with the piwi-interacting RNA (piRNA)-binding Piwi proteins, and such interactions are dependent on symmetrically dimethylated arginines (sDMAs) of Piwi proteins. Tudor-Piwi interactions are crucial and are conserved in the germ cells of sexually reproducing animals, including mammals. In the September 1, 2010, issue of Genes \& Development, Liu and colleagues (pp. 1876-1881) use a combination of genetics, biochemistry, and crystallography to uncover the molecular and structural details of how Tudor recognizes and binds the sDMAs of the Piwi protein Aubergine.
\end{abstract}

It has long been known that, in Drosophila as well as many other species with morphologically asymmetrical oocytes, primordial germ cell (PGC) specification and maintenance in the progeny is dictated by maternally deposited determinants accumulated in the cytoplasm of the posterior end of the oocyte, which is known as germ or pole plasm (Ephrussi and Lehmann 1992; Mahowald 2001). In a series of classical experiments, the germ plasm was shown to be necessary and sufficient to induce the formation of a germline (Ephrussi and Lehmann 1992; Mahowald 2001). Many of the components of the germ plasm (RNA and proteins) are synthesized by the nurse cells, and accumulate during oogenesis in a stepwise fashion (Dansereau and Lasko 2008). Among these are the products of the genes vas (Vasa, a DEAD-box RNA helicase) (Hay et al. 1988; Lasko and Ashburner 1988), tud (Tudor, the founding member of the homonymous family) (Boswell and Mahowald 1985), aub (Aubergine, a Piwi protein) (Schupbach and Wieschaus 1991; Harris and Macdonald 2001), csul (Capsuleen, a methyltransferase)

[Keywords: Tudor; Aubergine; Piwi; arginine methylation; structure] ${ }^{1}$ Corresponding author.

E-MAIL mourelaz@uphs.upenn.edu; FAX (215) 898-9969.

Article is online at http://www.genesdev.org/cgi/doi/10.1101/gad.1977010.
(Gonsalvez et al. 2006; Anne et al. 2007), and valois (cofactor of csul) (Anne and Mechler 2005)-most of which were identified by genetic screens for maternaleffect sterility. The progeny carrying such mutations is viable but does not form a germline, and in some cases the embryos show posterior defects; hence, these genes are called posterior-grandchildless (Strome and Lehmann 2007). Recent studies uncovered the relation that ties these genes together and also provided molecular explanations for the mutant phenotypes: Csul and its cofactor, Valois, symmetrically dimethylate arginines on Aubergine that are recognized and bound by Tudor (Kirino et al. 2009, 2010b; Nishida et al. 2009). The formation of the Tud-Aub complex is required for the two proteins to localize and contribute to the germ plasm formation (Nishida et al. 2009; Kirino et al. 2010b). PGC specification in mammals follows a different mechanism, but the functional network of these genes is conserved and is essential in males for proper spermatogenesis (Chuma et al. 2006; Reuter et al. 2009; Vagin et al. 2009; Vasileva et al. 2009; Wang et al. 2009; Arkov and Ramos 2010; Siomi et al. 2010).

Piwi proteins constitute a subclade of the Argonaute family, and bind to the extremely diverse class of piwiinteracting RNAs (piRNAs) (Klattenhoff and Theurkauf 2008; Ghildiyal and Zamore 2009). All Piwi mutants studied cause sterility due to the developmental arrest of gametogenesis or regeneration defects in planarians, where they are essential for neoblast maintenance (Thomson and Lin 2009). The most studied Piwi proteins are Drosophila Aub, Piwi, and Ago3, and mouse Mili, Miwi, and Miwi2 (Thomson and Lin 2009). Piwi proteins colocalize with tudor domain-containing proteins in characteristic electron-dense, nonmembranous, cytoplasmic structures called germ or polar granules, which often contact mitochondria and/or assemble perinuclearly (Arkov and Ramos 2010). In Drosophila ovaries, a germ cell-specific organelle called the nuage is formed around the nucleus of the nurse cells, and germ granules containing Aub are located at the posterior pole of the oocyte. In mouse spermatocytes, Piwi proteins associate with various Tdrds (Tudor domain-containing proteins) during the formation of intermitochondrial cement (Mili/Tdrd1) (Wang et al. 2009) and chromatoid bodies (Miwi-Mili/ Tdrd6) (Vagin et al. 2009; Vasileva et al. 2009). 
piRNAs and Piwi proteins also have a well-documented role in retrotransposon silencing (Vagin et al. 2006; Aravin et al. 2007; Brennecke et al. 2007; Gunawardane et al. 2007; Shoji et al. 2009). Many Piwi/piRNA ribonucleoproteins (piRNPs) target complementary, active mobile elements and cleave them. The transposon remnants are processed as secondary piRNAs, which in turn are used for the feedback processing of primary, anti-transposon piRNAs. This adaptive mechanism has been termed the ping-pong amplification loop (Brennecke et al. 2007; Gunawardane et al. 2007). Moreover, Piwi proteins are required for transposon taming at the DNA level by facilitating suppressive DNA methylation during epigenetic reprogramming of the developing germline (Aravin et al. 2007; Kuramochi-Miyagawa et al. 2008). However, large subpopulations of piRNAs are not related to repeat elements, and their function remains unknown. Despite recent progress, the biogenesis of primary piRNAs, their exact mode of function, and their full RNA target spectrum remain largely unclear. Also, a dynamic variation in the protein complement, form, and localization of germ granules is observed, which implies that protein-protein and RNA-protein interactions can be modulated during RNP restructuring (Aravin et al. 2009; Chen et al. 2009; Wang et al. 2009; Vagin et al. 2009; Vasileva et al. 2009). To proceed from observation to comprehension, a mechanistic understanding of Piwi-Tudor associations in the variety of germ granules and their functional repercussions is necessary. The study by Liu et al. (2010) in the September 1, 2010, issue of Genes \& Development is a decisive step toward this goal.

Tudor is a $285-\mathrm{kDa}$ protein harboring 11 homonymous domains (Thomson and Lasko 2005). Previous work (Arkov et al. 2006) established that a truncation mutant carrying only domains 7-11 is able to rescue Aub localization at the germ plasm and germline formation in a tud-null background. Those domains were found to interact with Aub (Kirino et al. 2010b). Liu et al. (2010) now focus on the tud7-11 transgene and perform an elegant mutation study in an effort to identify a single tud domain within the transgene responsible for Aub association. They sequentially mutate a pair of conserved aromatic amino acid residues in each one of the predicted tud domains of tud7-11, and probe Aub localization and germ cell formation. These mutations are expected to precisely disrupt symmetrically dimethylated arginine (sDMA) binding (Sprangers et al. 2003; Friberg et al. 2009) without significantly affecting overall structure. Strikingly, only domain 8 appears to be unnecessary for these functions, with the rest being indispensable. This result suggests Tudor-Aubergine binding of complex stoichiometry, and it will be interesting to see in subsequent studies whether tud domains bind their targets independently or synergistically.

Liu et al. (2010) proceeded to the crystallization of a polypeptide (amino acids 2344-2515) encompassing tudor domain 11-thus termed extended (eTud11) — in the absence and presence of Aub peptides (amino acids 6-18) carrying a single sDMA (R13 or R15) (Fig. 3 in Liu et al. 2010). The canonical tud domain spans residues 23902446, (one-third of the overall size of eTud11). It is most interesting that the resolved structures depict eTud11 folding into a single compact structure, with the flanking $\mathrm{N}$ and $\mathrm{C}$ termini interlocked in an oligonucleotidebinding $(\mathrm{OB})$ fold at an off-center position and the tud domain to the side, connected to the OB fold via a helix at its $\mathrm{N}$ terminus and a small helix-linker loop at its $\mathrm{C}$ terminus (Fig. 3 in Liu et al. 2010). This structure is surprisingly similar (root-mean-square deviation: 1.84 ) to the extended tudor domain of the Tudor SN/p100 protein fold reported by Sattler's team (Friberg et al. 2009), which was also shown to preferentially bind sDMAs. The tud 11 domain forms a barrel-like structure consisting of four $\beta$ strands, and can be superimposed to Tud SN and the tud domain of the Survival of Motor Neurons (SMN) protein (Selenko et al. 2001; Sprangers et al. 2003), also with minimal deviation (Fig. 3 in Liu et al. 2010).

The structure within the tud domain that actually contacts the sDMA has been fittingly described as an aromatic cage. In eTud11, this cage is composed of four aromatic residues (Phe2403, Tyr2410, Phe2427, and Phe2430), with their benzene rings arranged as the right, back, upper, and left faces of an hexahedron. The sDMA enters from the front and lower side, and its guanidine group is stacked in parallel to the right and left faces of the cage, stabilized by both hydrophobic and cation- $\pi$ interactions. The modified residue is secured in place by what looks like a latch: An asparagine residue (Asn2432) protruding from the upper rim of the cage-like structure makes a hydrogen bond with its amide carbonyl and the outward amino group of the sDMA. Mutating Asn2432 to alanine results into a dramatic decrease in the affinity of the tud domain for the methylated peptide. Given its "gatekeeper" role in sDMA binding, it is interesting to note that this residue displays various degrees of conservation, even within tudor proteins with germline functions, such as Drosophila Tudor (present in eight out of 11 domains), Krimper (one out of two) (Lim and Kai 2007), and Tejas (zero out of one) (Patil and Kai 2010), and mouse Tdrd6 (six out of seven), Tdrd1 (four out of four), and RNF17/Tdrd4 (two out of five) (Pan et al. 2005).

The study by Liu et al. (2010) also provides important evidence on the role of secondary sites of interaction involving Aub residues of the RG/RA motif, and structural elements other than the tud domain. Such interactions stabilize the peptide backbone that is positioned in parallel to the $\mathrm{N}$-terminal helix $(\alpha \mathrm{A})$ connecting the tud domain to the OB fold, and facilitate the exposure of the methylated side chain to the primary site of interaction: the aromatic cage. Glycine at position -1 relative to the sDMA and arginine at position -2 contact two residues at the $\alpha$ A helix. The latter is a glutamic acid (E2374) and forms a hydrogen bond with a side chain amino group of the -2 arginine. Moreover, in the resolved structure of the Aub peptide methylated at R15, R11 (position -4) makes multiple contacts with OB-fold residues, two of which are hydrogen bonds, through its two amino groups. It is assumed that these contacts can "sense" the methylation state of the Arg11. Unfortunately, no evidence is provided for interactions that are formed with Aub residues outside the RG motif due to crystal disorder. 
Such evidence would help us understand the interactions that the tudor module uses to distinguish its cognate partner between proteins carrying similar RG/RA motifs.

The two most conserved residues in $>1000$ tudor domains catalogued in the pfam protein family database (Finn et al. 2010) are also present in eTud11. These are an arginine (R2411) and an aspartate (D2429) located on the external surface of the tud $\beta$ barrel that form charged interactions with each other through their side chains. Asp2429 lies between two of the aromatic residues forming the sDMA-binding cage, but faces in an opposite direction. In addition to the charged interaction, the R-D pair makes multiple contacts with the C-terminal small helix-linker loop that connects the tud domain with the OB fold. These interactions form a network that consolidates the tud domain with the rest of the module and stabilizes the overall structure. Intriguingly, both residues are substituted in the tud domain of SMN from several species (Battle et al. 2006). The importance of this interaction is depicted in mutants $t_{u} d^{\mathrm{A} 36}$ and $t u d^{\mathrm{B} 42}$ (Arkov et al. 2006), both of which carry a mutation of the respective arginine (of the R-D pair described above) in domains tud 1 and tud10, respectively. tud ${ }^{\mathrm{A} 36}$ has a severe defect in germ granule morphology, but, perhaps, not surprisingly, can still form a germline, as the tud 1 domain was shown to be dispensable for this function (Arkov et al. 2006). $\mathrm{tud}^{\mathrm{B} 42}$ shows a complete loss of germ cells, underlining the importance of this tud domain for Aub binding and subsequent germline formation (Arkov et al. 2006).

Liu et al. (2010) conclude by making an intriguing observation: The sequence motif YR-D(F/Y)GN appears to be conserved in the Piwi-binding extended Tudor domain, making it a good starting point for designing subsequent experiments. Remarkably, this motif occurs in all five tudor domains of the tud7-11 truncation mutant, which was shown repeatedly to mediate Aub binding, but is found in only two of the other six tudor domains, which are dispensable for Aub binding.

In summary, this study uncovers discrete roles of specific amino acid residues for the formation of the aromatic cage within the tudor domain responsible for the binding of sDMA: as auxiliary attaching sites for the Aub backbone chain that possibly also play a role in recognizing the sDMA, and thereby drive it toward the tud domain and the sDMA-binding cage; and for the stabilization of the overall fold of the tud domain and surrounding structures. Furthermore, a signature motif for germline tudor domains has been put forward that provides a testable prediction for consideration in subsequent studies. Moreover, as illustrated in the examples described above, we can now integrate structural and genetic data, and draw several fascinating conclusions on the structure and function relationships of the multiple tudor domains.

There are still many important questions to be answered. Tudor has a role in abdominal patterning of the developing embryo, and, strikingly, different tudor domains can rescue a strong loss-of-function mutant in a similar way (Arkov et al. 2006). This redundancy is also implied in mice, where many Tdrds harbor multiple tudor domains. Is this an adaptation important for struc- turing the large RNPs of the germ plasm? Piwi proteins are the only examples of proteins that have been shown to interact via their sDMAs with germline Tdrds. Are there other sDMA-modified proteins that interact with specific germline Tdrds? For example, it was shown recently that Vasa carries both sDMAs and aDMAs (asymmetrically dimethylated arginines), the former of which are produced by Capsuleen (Kirino et al. 2010a). Although the sDMAs of Vasa appear dispensable for its interaction with Tudor (Kirino et al. 2010a), it is unknown whether they are required for interaction with other Tdrds. Additional Drosophila proteins with important germline functions carry tudor domains (Tejas, Spindle-E, and Krimper), but no methylated binding partner has been specified for them yet. Finally, the exact molecular function of the Piwi-Tudor interactions is unclear. It looks like there will be more interesting stories on tudors and their binding partners in the near future.

\section{Acknowledgments}

This work was supported by a Human Frontier Science Program Long-Term Fellowship to Y.K., and NIH grant GM0720777 to Z.M.

\section{References}

Anne J, Mechler BM. 2005. Valois, a component of the nuage and pole plasm, is involved in assembly of these structures, and binds to Tudor and the methyltransferase Capsuleen. Development 132: 2167-2177.

Anne J, Ollo R, Ephrussi A, Mechler BM. 2007. Arginine methyltransferase Capsuleen is essential for methylation of spliceosomal Sm proteins and germ cell formation in Drosophila. Development 134: 137-146.

Aravin AA, Sachidanandam R, Girard A, Fejes-Toth K, Hannon GJ. 2007. Developmentally regulated piRNA clusters implicate MILI in transposon control. Science 316: 744-747.

Aravin AA, van der Heijden GW, Castaneda J, Vagin VV, Hannon GJ, Bortvin A. 2009. Cytoplasmic compartmentalization of the fetal piRNA pathway in mice. PLoS Genet 5: e1000764. doi: 10.1371/journal.pgen.1000764.

Arkov AL, Ramos A. 2010. Building RNA-protein granules: Insight from the germline. Trends Cell Biol. 20: 482-490

Arkov AL, Wang JY, Ramos A, Lehmann R. 2006. The role of Tudor domains in germline development and polar granule architecture. Development 133: 4053-4062.

Battle DJ, Kasim M, Yong J, Lotti F, Lau CK, Mouaikel J, Zhang Z, Han K, Wan L, Dreyfuss G. 2006. The SMN complex: An assembly machine for RNPs. Cold Spring Harb Symp Quant Biol 71: 313-320.

Boswell RE, Mahowald AP. 1985. tudor, a gene required for assembly of the germ plasm in Drosophila melanogaster. Cell 43: 97-104.

Brennecke I, Aravin AA, Stark A, Dus M, Kellis M, Sachidanandam R, Hannon GJ. 2007. Discrete small RNA-generating loci as master regulators of transposon activity in Drosophila. Cell 128: 1089-1103.

Chen C, Jin J, James DA, Adams-Cioaba MA, Park JG, Guo Y, Tenaglia E, Xu C, Gish G, Min J, et al. 2009. Mouse Piwi interactome identifies binding mechanism of Tdrkh Tudor domain to arginine methylated Miwi. Proc Natl Acad Sci 106: 20336-20341. 
Chuma S, Hosokawa M, Kitamura K, Kasai S, Fujioka M, Hiyoshi M, Takamune K, Noce T, Nakatsuji N. 2006. Tdrd1/Mtr-1, a tudor-related gene, is essential for male germ-cell differentiation and nuage/germinal granule formation in mice. Proc Natl Acad Sci 103: 15894-15899.

Dansereau DA, Lasko P. 2008. The development of germline stem cells in Drosophila. Methods Mol Biol 450: 3-26.

Ephrussi A, Lehmann R. 1992. Induction of germ cell formation by oskar. Nature 358: 387-392.

Finn RD, Mistry J, Tate J, Coggill P, Heger A, Pollington JE, Gavin OL, Gunasekaran P, Ceric G, Forslund K, et al. 2010. The Pfam protein families database. Nucleic Acids Res 38: D211-D222. doi: 10.1093/nar/gkp985.

Friberg A, Corsini L, Mourao A, Sattler M. 2009. Structure and ligand binding of the extended Tudor domain of D. melanogaster Tudor-SN. J Mol Biol 387: 921-934.

Ghildiyal M, Zamore PD. 2009. Small silencing RNAs: An expanding universe. Nat Rev Genet 10: 94-108.

Gonsalvez GB, Rajendra TK, Tian L, Matera AG. 2006. The Smprotein methyltransferase, dart5, is essential for germ-cell specification and maintenance. Curr Biol 16: 1077-1089.

Gunawardane LS, Saito K, Nishida KM, Miyoshi K, Kawamura Y, Nagami T, Siomi H, Siomi MC. 2007. A slicer-mediated mechanism for repeat-associated siRNA $5^{\prime}$ end formation in Drosophila. Science 315: 1587-1590.

Harris AN, Macdonald PM. 2001. Aubergine encodes a Drosophila polar granule component required for pole cell formation and related to eIF2C. Development 128: 2823-2832.

Hay B, Jan LY, Jan YN. 1988. A protein component of Drosophila polar granules is encoded by vasa and has extensive sequence similarity to ATP-dependent helicases. Cell 55: 577-587.

Kirino Y, Kim N, de Planell-Saguer M, Khandros E, Chiorean S, Klein PS, Rigoutsos I, Jongens TA, Mourelatos Z. 2009. Arginine methylation of Piwi proteins catalysed by dPRMT5 is required for Ago3 and Aub stability. Nat Cell Biol 11: 652-658.

Kirino Y, Vourekas A, Kim N, de Lima Alves F, Rappsilber J, Klein PS, Jongens TA, Mourelatos Z. 2010a. Arginine methylation of vasa protein is conserved across phyla. J Biol Chem 285: 8148-8154.

Kirino Y, Vourekas A, Sayed N, de Lima Alves F, Thomson T, Lasko P, Rappsilber J, Jongens TA, Mourelatos Z. 2010b. Arginine methylation of Aubergine mediates Tudor binding and germ plasm localization. RNA 16: 70-78.

Klattenhoff C, Theurkauf W. 2008. Biogenesis and germline functions of piRNAs. Development 135: 3-9.

Kuramochi-Miyagawa S, Watanabe $\mathrm{T}$, Gotoh K, Totoki $\mathrm{Y}$, Toyoda A, Ikawa M, Asada N, Kojima K, Yamaguchi Y, Ijiri TW, et al. 2008. DNA methylation of retrotransposon genes is regulated by Piwi family members MILI and MIWI2 in murine fetal testes. Genes Dev 22: 908-917.

Lasko PF, Ashburner M. 1988. The product of the Drosophila gene vasa is very similar to eukaryotic initiation factor-4A. Nature 335: 611-617.

Lim AK, Kai T. 2007. Unique germ-line organelle, nuage, functions to repress selfish genetic elements in Drosophila melanogaster. Proc Natl Acad Sci 104: 6714-6719.

Liu H, Wang J-YS, Huang Y, Li Z, Gong W, Lehmann R, Xu R-M. 2010. Structural basis for methylarginine-dependent recognition of Aubergine by Tudor. Genes Dev 24: 1876-1881.

Mahowald AP. 2001. Assembly of the Drosophila germ plasm. Int Rev Cytol 203: 187-213.

Nishida KM, Okada TN, Kawamura T, Mituyama T, Kawamura Y, Inagaki S, Huang H, Chen D, Kodama T, Siomi H, et al. 2009. Functional involvement of Tudor and dPRMT5 in the piRNA processing pathway in Drosophila germlines. EMBO J 28: 3820-3831.
Pan J, Goodheart M, Chuma S, Nakatsuji N, Page DC, Wang PJ. 2005. RNF17, a component of the mammalian germ cell nuage, is essential for spermiogenesis. Development 132: 4029-4039.

Patil VS, Kai T. 2010. Repression of retroelements in Drosophila germline via piRNA pathway by the Tudor domain protein Tejas. Curr Biol. 20: 724-730

Reuter M, Chuma S, Tanaka T, Franz T, Stark A, Pillai RS. 2009. Loss of the Mili-interacting Tudor domain-containing protein-1 activates transposons and alters the Mili-associated small RNA profile. Nat Struct Mol Biol 16: 639-646.

Schupbach T, Wieschaus E. 1991. Female sterile mutations on the second chromosome of Drosophila melanogaster. II. Mutations blocking oogenesis or altering egg morphology. Genetics 129: 1119-1136.

Selenko P, Sprangers R, Stier G, Buhler D, Fischer U, Sattler M. 2001. SMN tudor domain structure and its interaction with the Sm proteins. Nat Struct Biol 8: 27-31.

Shoji M, Tanaka T, Hosokawa M, Reuter M, Stark A, Kato Y, Kondoh G, Okawa K, Chujo T, Suzuki T, et al. 2009. The TDRD9-MIWI2 complex is essential for piRNA-mediated retrotransposon silencing in the mouse male germline. Dev Cell 17: 775-787.

Siomi MC, Mannen T, Siomi H. 2010. How does the royal family of Tudor rule the PIWI-interacting RNA pathway? Genes Dev 24: 636-646.

Sprangers R, Groves MR, Sinning I, Sattler M. 2003. Highresolution X-ray and NMR structures of the SMN Tudor domain: Conformational variation in the binding site for symmetrically dimethylated arginine residues. I Mol Biol 327: 507-520.

Strome S, Lehmann R. 2007. Germ versus soma decisions: Lessons from flies and worms. Science 316: 392-393.

Thomson T, Lasko P. 2005. Tudor and its domains: Germ cell formation from a Tudor perspective. Cell Res 15: 281-291.

Thomson T, Lin H. 2009. The biogenesis and function of PIWI proteins and piRNAs: Progress and prospect. Annu Rev Cell Dev Biol 25: 355-376.

Vagin VV, Sigova A, Li C, Seitz H, Gvozdev V, Zamore PD. 2006. A distinct small RNA pathway silences selfish genetic elements in the germline. Science 313: 320-324.

Vagin VV, Wohlschlegel J, Qu J, Jonsson Z, Huang X, Chuma S, Girard A, Sachidanandam R, Hannon GJ, Aravin AA. 2009. Proteomic analysis of murine Piwi proteins reveals a role for arginine methylation in specifying interaction with Tudor family members. Genes Dev 23: 1749-1762.

Vasileva A, Tiedau D, Firooznia A, Muller-Reichert T, Jessberger R. 2009. Tdrd6 is required for spermiogenesis, chromatoid body architecture, and regulation of miRNA expression. Curr Biol 19: 630-639.

Wang J, Saxe JP, Tanaka T, Chuma S, Lin H. 2009. Mili interacts with tudor domain-containing protein 1 in regulating spermatogenesis. Curr Biol 19: 640-644. 


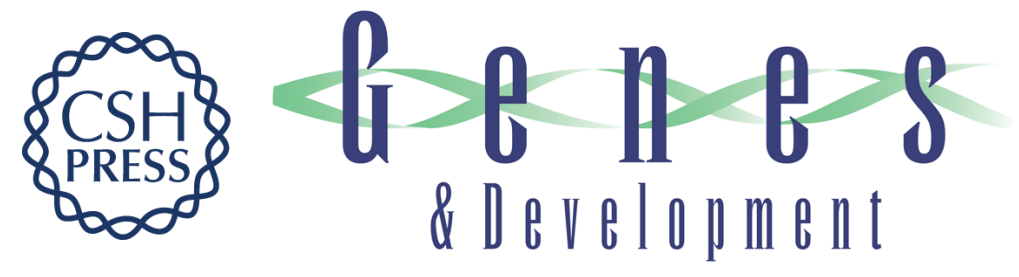

\section{Elective affinities: a Tudor-Aubergine tale of germline partnership}

Anastassios Vourekas, Yohei Kirino and Zissimos Mourelatos

Genes Dev. 2010, 24:

Access the most recent version at doi:10.1101/gad.1977010

Related Content Structural basis for methylarginine-dependent recognition of Aubergine by Tudor Haiping Liu, Ju-Yu S. Wang, Ying Huang, et al.

Genes Dev. September , 2010 24: 1876-1881

References This article cites 45 articles, 20 of which can be accessed free at: http://genesdev.cshlp.org/content/24/18/1963.full.html\#ref-list-1

Articles cited in:

http://genesdev.cshlp.org/content/24/18/1963.full.html\#related-urls

\section{License}

Email Alerting

Receive free email alerts when new articles cite this article - sign up in the box at the top Service

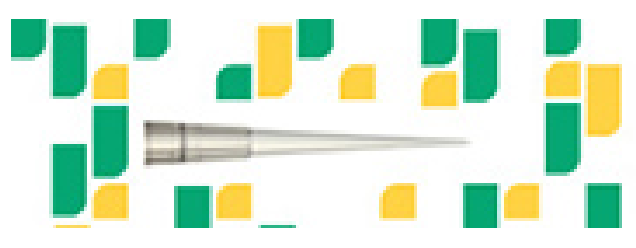

Focused on your science. 